\title{
Panorama de la infección por Listeria monocytogenes
}

\author{
Juan Pablo Rodríguez-Auad ${ }^{1}$
}

\section{Overview of Listeria monocytogenes infection}

Listeriosis is an uncommon but potentially serious infection caused by Listeria monocytogenes. The main route of transmission is through the consumption of contaminated food. It generally affects elderly people, pregnant women and immunosuppressed hosts, although cases are also seen in immunocompetent adults and children. Listeria monocytogenes is a short, anaerobic, non-spore-forming gram-positive bacillus that causes a narrow zone of hemolysis in blood agar. It is a facultative intracellular pathogen, and therefore it shows a complex pathogenesis. This bacterium has the ability to cross the intestinal barrier, the placenta and the blood-brain barrier producing gastroenteritis, maternal-fetal infections and meningoencephalitis. It is most commonly diagnosed from a positive culture of a sterile site. The treatment of choice includes the use of intravenous ampicillin alone or in combination with gentamicin.

Keywords: Listeriosis, meningoencephalitis, ampicillin, pediatrics.

Palabras clave: Listeriosis, meningoencefalitis, ampicilina, pediatría.

\section{Introducción}

$L$ isteria monocytogenes fue aislada por primera vez por E.G.D. Murray y cols. en 1926 durante una epidemia en animales, principalmente de cuyes y conejos. Originalmente fue llamada como Bacterium monocytogenes por el incremento de monocitos que se encontró en la sangre de los animales infectados; posteriormente fue nombrada Listerella hepatolytica, en honor a Joseph Lister, el pionero en el campo de la antisepsia y, finalmente en 1940, adoptó el nombre de Listeria monocytogenes ${ }^{1}$.

La listeriosis es una infección infrecuente pero potencialmente grave, causada por L. monocytogenes. Este microorganismo puede ser encontrado en el suelo, vegetación y en animales. La principal vía de transmisión es por el consumo de alimentos contaminados, aunque también se han visto casos de transmisión directa de animales a humanos y entre humanos. Afecta generalmente a personas mayores, mujeres embarazadas e inmunocomprometidos (incluyendo neonatos), aunque también se describen casos en adultos y niños inmunocompetentes ${ }^{2-5}$. A diferencia de otras infecciones asociadas al consumo de alimentos, la listeriosis presenta una elevada tasa de mortalidad (20-30\%) $)^{6,7}$.

En infecciones neonatales, L. monocytogenes puede ser transmitida de la madre al hijo in utero o durante el paso a través del canal del parto ${ }^{8}$. Hay también reportes de transmisión nosocomial por material contaminado y alimentos contaminados que ingresaron al hospital ${ }^{9}$. Esta bacteria puede sobrevivir al proceso de acidificación de las comidas y puede continuar su multiplicación len- tamente a bajas temperaturas, pudiendo crecer incluso en alimentos adecuadamente refrigerados. Es capaz de producir biopelícula en alimentos, crecer a temperaturas de refrigeración, resistir condiciones adversas de $\mathrm{pH}$ y altas concentraciones de $\mathrm{NaCl}^{10}$.

\section{Etiología}

Listeria monocytogenes es un bacilo grampositivo corto, anaerobio facultativo, no formador de esporas, móvil, que provoca una zona angosta de hemólisis en agar sangre. De las seis especies del género Listeria: Listeria monocytogenes, Listeria innocua, Listeria ivanovii, Listeria seeligeri, Listeria welshimeri y L.marthii, sólo L. monocytogenes es patogénica para el hombre. De los 13 serotipos de L. monocytogenes, los serotipos $1 / 2 \mathrm{a}, 1 / 2 \mathrm{~b}$ y $4 \mathrm{~b}$ son los responsables de la mayoría de los casos clínicos ${ }^{11}$.

\section{Epidemiología}

Esta bacteria se distribuye ampliamente en la naturaleza, es causa importante de zoonosis, en especial, en animales de rebaño. Se aísla a partir de aguas residuales de ensilajes y del suelo, donde alcanza a vivir más de 295 días ${ }^{12}$. Brotes de infecciones por L. monocytogenes se han observado en varios países por consumo de quesos blandos y de otros alimentos ${ }^{13,14}$. Casos esporádicos de listeriosis están asociados a factores de riesgo dietéticos, que incluyen el consumo de alimentos crudos o mal cocidos y fiambres ${ }^{15}$. Los principales alimentos incriminados son leche, quesos blandos no pasteurizados ${ }^{16}$, carnes preparadas listas para
'Servicio de Infectología. Hospital del Niño Dr. Ovidio Aliaga Uría. La Paz, Bolivia.

El autor no presenta conflictos de intereses.

Fuente de financiamiento: Autofinanciado.

Recibido: 13 de septiembre de 2017

Aceptado: 12 de noviembre de 2018

Correspondencia a: Juan Pablo Rodríguez-Auad juparodau@hotmail.com 
consumir (salchichas, carnes exóticas y paté); ave de corral y pescados mal cocidos, verduras crudas no lavadas e inclusive frutas contaminadas ${ }^{17,18}$. El uso de antiácidos (antagonistas de los receptores $\mathrm{H}_{2}$ ) se ha asociado también a un mayor riesgo de adquirir la infección ${ }^{19}$.

En un estudio efectuado en Brasil, se detectó L. monocytogenes en 3,1\% de verduras listas para consumir ${ }^{20}$. En otro estudio realizado en México, se encontró éste y otros patógenos en dos tipos de queso de consumo habitual ${ }^{21}$.

Entre 1 y $10 \%$ de la población es un portador fecal de $L$. monocytogenes ${ }^{22}$. La portación silenciosa de $L$. monocytogenes durante la gestación puede ocasionar enfermedad neonatal esporádica, por vía transplacentaria, vía ascendente o por exposición durante el parto.

En la mujer embarazada, la infección por L. monocytogenes ocurre 13 a 20 veces con mayor frecuencia que en la población general ${ }^{23}$, con una incidencia de listeriosis en el embarazo de 12 por 100.000 comparado con la incidencia de 0,7 por 100.000 en la población general ${ }^{24}$.

La infección neonatal tardía se puede deber a la adquisición del microorganismo durante el pasaje por el canal del parto o de fuentes ambientales, por ingestión de la bacteria, seguida de su diseminación hematógena desde el intestino ${ }^{25}$. La incidencia neonatal de listeriosis varía según los países y puede ir de 0,61 a 13 por 100.000 nacidos vivos, y es una de las causas más comunes de meningitis neonatal ${ }^{8,25,26}$. En un hospital de tercer nivel en México, se ha reportado una incidencia de 1 por 1.500 nacidos vivos ${ }^{27}$.

Las manifestaciones clínicas de la listeriosis neonatal son similares a la de Streptococcus agalactiae (Streptococcus $\beta$ hemolítico grupo B), y su tasa de mortalidad es elevada $(20-60 \%)^{24,28}$. Aunque se dice que la listeriosis es 100 a 1.000 veces más frecuente en pacientes en etapa SIDA (síndrome de inmunodeficiencia adquirida) ${ }^{8}$, llama la atención que no se vean muchos casos en esta población ${ }^{29}$. Se han observado brotes nosocomiales en salas de neonatología, y en los últimos años ha habido un incremento de casos en países europeos ${ }^{30}$. Varios estudios en Latinoamérica ${ }^{31-33}$ coinciden en que existe un bajo reporte de casos y una subestimación del problema; sin embargo, se espera que, por las condiciones sanitarias de muchos países, la frecuencia y letalidad sea elevada en comparación con países desarrollados ${ }^{32}$; por ejemplo, en un hospital del Perú, L. monocytogenes ocupa el primer lugar como causa de meningitis neonatal ${ }^{31}$.

Globalmente, se ha estimado que L. monocytogenes causa más de 2.500 infecciones y 500 muertes anuales en los Estados Unidos de América ${ }^{23}$.

\section{Patogenia}

Listeria monocytogenes es un patógeno intracelular facultativo cuya expresión de factores de virulencia están controlados por múltiples mecanismos regulados por el $\mathrm{ARN}^{34}$. Su patogenia íntima es compleja e incluye: adhesión a la célula hospedera e invasión (internalización), su fuga de las vacuolas fagocíticas, multiplicación intracelular y movilidad que le lleva propagarse de célula a célula ${ }^{13,35}$.

Una vez que un alimento contaminado es ingerido, Listeria es fagocitada por las células gastrointestinales -se ha visto que esta bacteria causa su propia internalización a células no fagocíticas $-^{36}$, donde evita ser destruida porque produce una hemolisina (listeriolisina) y fosfolipasas que degradan la membrana del fagosoma evitando su digestión intracelular. Siendo fagocitada por los macrófagos, escapa hacia su citoplasma donde se multiplica y prolifera para luego traspasar a las células adyacentes. A través de estos pasos, Listeria se puede multiplicar y diseminarse en el organismo sin haber sido expuesta a los anticuerpos, neutrófilos o antimicrobianos del líquido extracelular ${ }^{19}$. Una vez que cruza la barrera intestinal, se disemina a la sangre y a los ganglios mesentéricos. De allí migra hacia el hígado y al bazo para multiplicarse dentro de los macrófagos hepáticos y esplénicos, respectivamente, o en las células epiteliales del parénquima. Si en este momento no actúa una eficiente respuesta del sistema inmune innato, la bacteria es capaz de evadir la respuesta inmune y continuar su multiplicación en el organismo ${ }^{35,37}$. Este ciclo celular protege a esta bacteria de la inmunidad humoral y es responsable, en cambio, de la activación de inmunidad mediada por células $\mathrm{T}^{13}$. La resistencia a la infección depende, entonces, de la inmunidad celular. Las células $\mathrm{T}$ citotóxicas $(\mathrm{CD} 8+)$ reconocen y lisan células infectadas mientras las bacterias extracelulares son atacadas por fagocitos activados circulantes. En pacientes con disminución de la inmunidad celular no se logra controlar la multiplicación bacteriana y se produce bacteriemia e invasión de órganos, especialmente del cerebro y del útero grávido. Los individuos con disfunción de las células $\mathrm{T}$ tienen mayor riesgo de contraer la enfermedad ${ }^{38}$. Sin embargo, la enfermedad invasora puede ocurrir, también, en personas sin compromiso inmunológico alguno ${ }^{39}$. Estudios placentarios han demostrado que L. monocytogenes puede invadir la placenta, por vía directa, del sincicio-trofoblasto o a través de la propagación de célula a célula de la decidua o de bacterias localizadas dentro de los leucocitos maternos a nivel del citotrofoblasto extravelloso para luego ingresar al espacio intrauterino.

Varios factores de virulencia de L. monocytogenes han sido implicados en la infección placentaria; por ejemplo, las internalinas (Inl) A, InlB e InlP han demostrado contribuir a la invasión de células del trofoblasto, aunque muchos detalles sobre la infección vertical son desconocidos debido a las restricciones de efectuar estudios en humanos y a los distintos cambios placentarios que ocurren durante la gestación ${ }^{40,39}$. Atravesada la barrera 
materno-fetal, L. monocytogenes puede causar aborto espontáneo, muerte fetal intrauterina, parto prematuro e infección fetal diseminada con muerte fetal y neonatal, lo que ocurre en aproximadamente 20 a $60 \%$ de los casos. La infección puede ocurrir en cualquier momento durante el embarazo, pero con mayor frecuencia se detecta en el tercer trimestre ${ }^{39}$.

El tiempo promedio de incubación de la listeriosis es de 3 semanas $^{41}$, con un rango que puede ir de 3 a 70 días $^{39}$, aunque esto dependerá de las características del paciente. Por ejemplo, el período de incubación para enfermedad invasora es mayor en mujeres embarazadas ( 2 a 4 semanas) que en no embarazadas ( 1 a 14 días). El período de incubación para cuadros de gastroenteritis febriles autolimitadas seguida de la ingesta de grandes inóculos es de $24 \mathrm{~h}$, durando la enfermedad en estos casos 2 a 3 días $^{42}$.

La investigación sobre la patogenia de esta bacteria ha proporcionado información considerable sobre cómo las bacterias patógenas se adaptan a hospederos mamíferos, invaden a las células eucariotas, se mueven en el medio intracelular, interfieren con las funciones de la célula hospedera y se diseminan dentro de los tejidos ${ }^{33}$. Debido a sus numerosas características patogénicas y sobre la respuesta inmune, L. monocytogenes está siendo investigada como un vector en la inmunoterapia contra el cáncer ${ }^{43}$.

\section{Manifestaciones clínicas}

Listeria monocytogenes causa dos formas de listeriosis: gastrointestinal no invasora y listeriosis invasora. En individuos inmunocompetentes, la listeriosis no invasora se presenta como un cuadro típico de gastroenteritis febril (fiebre y diarrea). Puede comprometer diversos órganos teniendo particular predilección por el sistema nervioso central (SNC) ${ }^{44}$. En pacientes inmunosuprimidos, la listeriosis se puede manifestar como un cuadro de septicemia o meningoencefalitis. En la Figura 1 se observa la imagen tomográfica de un cuadro letal de meningoencefalitis en una adolescente de 14 años de edad con tratamiento inmunosupresor que presentó cefalea, fiebre y somnolencia, con el antecedente de haber presentado deposiciones líquidas una semana previa. Se identificó bacilos grampositivos en el LCR y en el cultivo del mismo desarrolló L. monocytogenes. La evolución fue tórpida y la paciente falleció pese al manejo antimicrobiano apropiado y de soporte intenso. Como en este caso descrito, los casos de meningoencefalitis se presentan generalmente con fiebre, cefalea intensa, náuseas, vómitos y signos de irritación meníngea. Cuadros de romboencefalitis que involucran el tronco cerebral son formas inusuales de presentación de la listeriosis ${ }^{45}$.

La listeriosis durante el embarazo es una seria amenaza para el feto; un tercio de los casos de transmisión

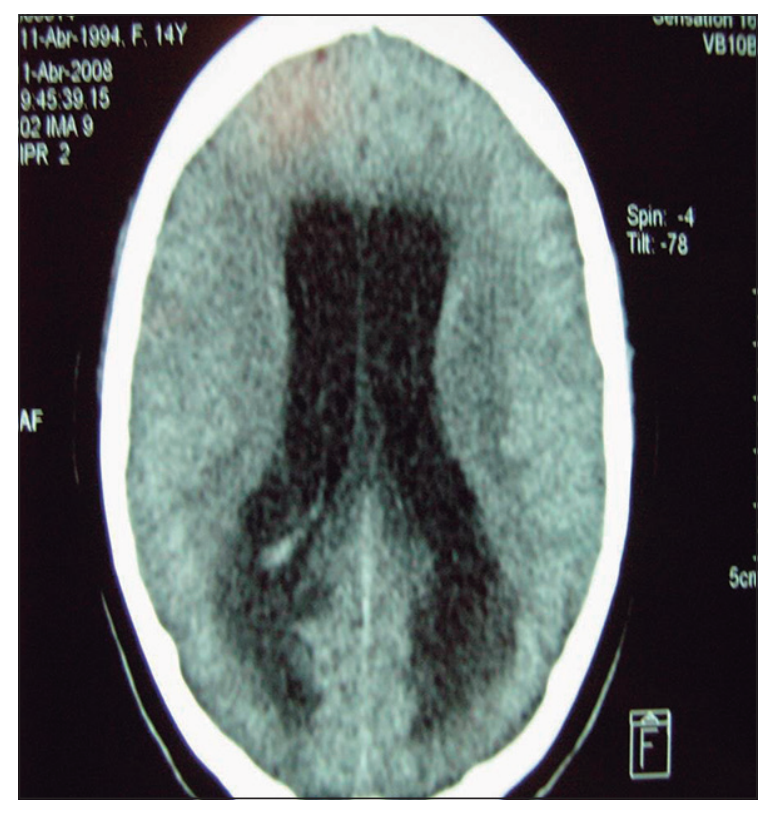

Figura 1. Hidrocefalia secundaria a meningoencefalitis por L. monocytogenes en una adolescente de 14 años de edad con diagnóstico de lupus eritematoso sistémico en tratamiento inmunosupresor.

materno-fetal confirmados por cultivo se resuelven como un aborto o mortinato. La gran mayoría de infecciones ocurre durante el tercer trimestre de embarazo, etapa en que la inmunidad de células T está más afectada ${ }^{23,8}$. Las mujeres embarazadas pueden desarrollar el cuadro de forma asintomática, con síntomas leves, sin fiebre, que son inespecíficos (malestar general, cefalea, síntomas digestivos y dorsalgia) o pueden presentar un cuadro febril con o sin síntomas gastrointestinales ${ }^{23}$.

La listeriosis neonatal se divide en dos formas clínicas: inicio temprano (primera semana de vida, $<7$ días) e inicio tardío ( $>7$ días). En la infección neonatal de inicio temprano los síntomas aparecen generalmente al primer o segundo día de vida, pudiendo el neonato presentar al nacimiento meconio, cianosis, apnea y neumonía ${ }^{8}$. También se puede observar un exantema eritematoso con pequeños nódulos pálidos ("granulomatosis infantoséptica"), mientras que en las infecciones de inicio tardío, la manifestación más frecuente es una meningitis, donde la fiebre e irritabilidad son los datos clínicos predominantes $^{46,8}$. El nacimiento de un feto prematuro infectado se asocia a una mortalidad infantil de 50 a $90 \%{ }^{13}$.

Fuera del período neonatal o del embarazo, las principales manifestaciones clínicas son: meningitis o infección del parénquima cerebral. Estos cuadros se presentan sobre todo en pacientes inmunosuprimidos (receptores de trasplantes, SIDA, enfermedad maligna hematológica o inmunosupresión por corticosteroides), pacientes en hemodiálisis, pacientes con diabetes mellitus y en personas mayores, a partir de 50 años de edad d5, $47,48^{\text {. }}$.

Listeria monocytogenes puede producir una gran 
variedad de infecciones localizadas; se han presentado casos de conjuntivitis, infecciones de piel, linfadenitis, abscesos hepáticos, abscesos cerebrales, peritonitis, abscesos esplénicos, infecciones pleuro-pulmonares, osteomielitis, pericarditis y miocarditis, entre otros ${ }^{24}$. Se ha descrito también infección relacionada a catéter de derivación ventrículo-peritoneal, pudiendo esta bacteria formar y sobrevivir dentro de la biopelícula en los dispositivos médicos ${ }^{49,50}$.

\section{Diagnóstico}

Se diagnostica, generalmente, una listeriosis a partir de un cultivo positivo de un sitio estéril. Se puede cultivar L. monocytogenes de muestras clínicas como sangre, líquido cefalorraquídeo (LCR) (Figura 2), líquido amniótico, placenta, meconio, lavado gástrico o hisopado ótico en los recién nacidos, y otras muestras de fluidos infectados (líquido sinovial, pleural o pericárdico). Se aísla L. monocytogenes de la sangre en $40-75 \%$ de los casos de meningitis causada por este microorganismo. La bacteria crece bien en agar sangre a $35^{\circ} \mathrm{C}$, tras 18 a $24 \mathrm{~h}$ de incubación en aerobiosis, formando pequeñas colonias redondeadas con $\beta$-hemólisis ${ }^{22}$. Se ha desarrollado un nuevo caldo enriquecido para su aislamiento desde alimentos con buenos resultados ${ }^{51}$. Las muestras de heces deben ser enriquecidas selectivamente para Listeria antes de ser sembradas en medios de cultivos selectivos. La tinción de Gram también es útil para revelar la presencia del microorganismo (Figura 3). Las muestras en las que más se ha aplicado esta tinción son: aspirado gástrico, tejido placentario, biopsias del exantema de la infección de comienzo temprano, LCR y líquido amniótico. En los

Figura 2. Crecimiento de L. monocytogenes en agar sangre de muestra de LCR de paciente de la Figura 1, obsérvese la hemólisis que produce esta bacteria en el cultivo.

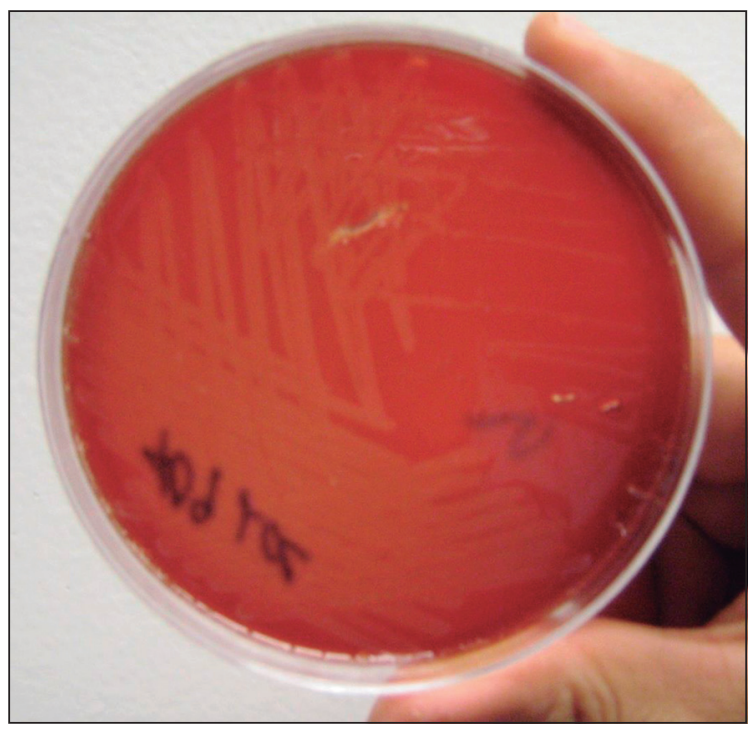

pacientes en quienes se reporta la presencia de "difteroides" en la tinción Gram o el cultivo, se debe sospechar e investigar listeriosis, ya que muchas veces se puede confundir Listeria con difteroides o estreptococos, dando el informe como contaminante o saprófito. Se debe avisar al laboratorio del diagnóstico de presunción clínica, para que los aislados de Listeria no se rechacen como contaminantes. Un aumento de monocitos en sangre periférica debe alertar al médico de la posibilidad de listeriosis ${ }^{13,41}$, si bien no existe un punto de corte en tal sentido. Se ha identificado un agente productor de monocitosis (APM) que estimula la producción de monocitos en la infección por L. monocytogenes ${ }^{8,52}$.

La prueba de reacción de polimerasa en cadena (RPC) es el único ensayo usado para la detección rápida de $L$. monocytogenes en muestras clínicas. Por otra parte, con fines de investigación epidemiológica, es fundamental intentar el aislamiento de la bacteria para los estudios y medidas de control posteriores.

Durante el embarazo es difícil hacer el diagnóstico de listeriosis, por lo que se debe considerar la realización de hemocultivos en cualquier mujer embarazada que presenta fiebre, especialmente si se acompaña de síntomas "gripales" y/o gastrointestinales. Si se ha realizado amniocentesis, es muy importante realizar la tinción Gram ya que ésta revela usualmente bacilos grampositivos ${ }^{23}$. Muestras vaginales o de heces no ayudan al diagnóstico ya que algunas mujeres son portadoras (asintomáticas) ${ }^{24}$.

\section{Tratamiento}

In vitro, L. monocytogenes es susceptible a una gran variedad de antimicrobianos, con excepción de fosfomi-

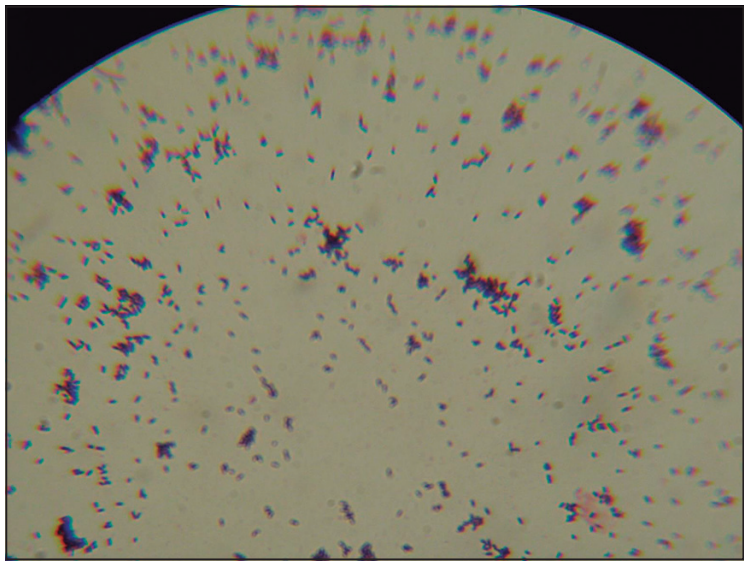

Figura 3. Tinción Gram de muestra de cultivo de LCR de paciente de la Figura 1, obsérvese la morfología característica de L. monocytogenes (bacilo corto grampositivo, no esporulado). 
cina, quinolonas de primera generación y cefalosporinas de tercera generación, susceptibilidad que no ha cambiado considerablemente en los últimos 35 años $^{53,54}$. Penicilina, ampicilina y amoxicilina se han usado más extensamente en casos de listeriosis. El tratamiento de elección incluye el uso de ampicilina intravenosa sola o en combinación con gentamicina, ya que esta última ha demostrado sinergia con ampicilina y menor riesgo de muerte usando el esquema combinado ${ }^{23,55-57}$; no hay estudios que indiquen el tiempo de uso de gentamicina, pero la experiencia del autor es mantener la sinergia por siete días, para luego continuar con ampicilina hasta completar el tratamiento, una vez que el cultivo es negativo y se ha verificado susceptibilidad a ampicilina. En casos de mala respuesta al tratamiento inicial se ha usado cotrimoxazol con buenos resultados ${ }^{58}$. Los antimicrobianos más efectivos (penicilina y ampicilina) tienen actividad bacteriostática contra L. monocytogenes, por lo que las propias defensas celulares del paciente juegan un rol fundamental contra esta bacteria. El tiempo de tratamiento para las infecciones invasoras sin meningitis es de 10-14 días. Para cuadros de meningitis por L. monocytogenes se recomienda tratamiento de 21 días $^{55,49}$, e inclusive este tiempo se puede extender en casos de endocarditis o romboencefalitis ${ }^{41}$. Se debe incrementar la dosis de ampicilina cuando se sospeche en meningitis, a $300 \mathrm{mg} / \mathrm{kg} /$ día (200-400 mg/ $\mathrm{kg} /$ día), fraccionada en 4 a 6 dosis diarias. En el recién nacido se ajustará la dosis y el intervalo de administración según su peso y días de vida.

Autores brasileños estudiaron muestras de pacientes con infección por L. monocytogenes aisladas desde 1970 a 2008, siendo $100 \%$ de éstas susceptibles a ampicilina ${ }^{59}$, lo mismo ocurrió en aislados a partir de alimentos en Colombia $^{60}$. Sin embargo, en México se ha reportado cepas multi-resistentes en muestras tomadas de pescados y agua de mar, que podrían ser una amenaza potencial para el ser humano ${ }^{61}$.

En la mujer embarazada, si bien algunos autores recomiendan diferir el tratamiento en casos asintomáticos o con síntomas leves sin fiebre, se debe recalcar que mientras en el período de gestación el cuadro puede ser leve o asintomático, la infección podría ser grave para el feto y para el recién nacido, incluyendo abortos, muerte fetal, sepsis y meningitis neonatal. Por eso, en las pacientes embarazadas con cuadro febril, con o sin síntomas gastrointestinales, con sospecha de exposición a L. monocytogenes sin otra causa que explique el cuadro febril, se deben solicitar los estudios diagnósticos (hemocultivo) e iniciar el manejo antibacteriano empírico correspondiente, con dosis altas de ampicilina por vía intravenosa ( $6 \mathrm{~g} /$ día $)$ por un período de 14 días $^{23,28}$. Incluso, mujeres embarazadas tratadas con dosis mayores de ampicilina (12 g/ día) por tres semanas en el primer trimestre de embarazo tuvieron buena respuesta a la misma llegando a tener un embarazo a término sin complicaciones ${ }^{62}$. El diagnóstico y tratamiento temprano en la mujer embarazada incrementa la posibilidad de sobrevida del recién nacido ${ }^{63}$.

Dado que Listeria no produce usualmente $\beta$-lactamasa, la adición de inhibidores de $\beta$-lactamasa resulta innecesaria. Para los pacientes alérgicos a penicilina, el uso de cotrimoxazol es una alternativa. Vancomicina ha sido usada también como tratamiento en casos de bacteriemia por Listeria; sin embargo, es poco efectiva para infecciones del SNC. En algunos casos, linezolid ha sido usado con éxito para el tratamiento de infecciones por Listeria ${ }^{64}$. Dado que L. monocytogenes se reproduce en el sistema reticuloendotelial y sobrevive en el medio intracelular al interior de los macrófagos, el uso de macrólidos y quinolonas podría favorecer el manejo de las bacterias intracelulares. Las quinolonas más recientes parecen ser de elevado valor para el tratamiento de listeriosis por su excelente penetración a los tejidos y a nivel celular y por ser rápidamente bactericidas; sin embargo, en la práctica no han demostrado los resultados esperados. Se ha propuesto a levofloxacina como terapia empírica para meningitis bacteriana que cubriría a Listeria, pero su uso tiene que ser evaluado en un mayor número de $\operatorname{casos}^{55}$. Moxifloxacina es un candidato prometedor ${ }^{65,66}$, aunque no hay ensayos clínicos controlados que apoyen su uso durante la gestación y tendría limitaciones por eventuales efectos deletéreos sobre el feto ${ }^{67}$. Las cefalosporinas no tienen actividad contra $L$. monocytogenes debido a su pobre o inexistente afinidad a las proteínas fijadoras de penicilina (PBP) 3 y 5 de Listeria $^{68}$. Sin embargo, debido al uso inadecuado de antimicrobianos y al uso de éstos en el mundo veterinario, se ha generado presión selectiva que ha llevado a la producción de cepas de Listeria resistentes a antimicrobianos usados con frecuencia para esta enfermedad ${ }^{69}$.

\section{Prevención}

Si bien la exposición a L. monocytogenes no se puede evitar completamente, una adecuada preparación y almacenamiento de los alimentos puede disminuir el riesgo de contraer la enfermedad. Normas generales para prevenir listeriosis incluyen: cocinar por completo los alimentos de origen animal; lavar las verduras crudas; separar las carnes crudas de verduras, alimentos no cocidos y alimentos listos para consumir; evitar productos lácteos no pasteurizados; lavarse las manos y lavar cuchillos y tablas empleados en preparar las comidas después del contacto con alimentos no cocidos. Las mujeres embarazadas y pacientes inmunocomprometidos deben evitar el consumo de leche cruda, quesos blandos no pasteurizados, carnes embutidas (fiambres), salchichas no cocidas adecuadamente, patés y otros picadillos de carne 
Tabla 1. Recomendaciones dietéticas para prevenir listeriosis ${ }^{41,71}$

Lavado y manejo de alimentos:

Enjuague los productos crudos, a fondo, con agua del grifo antes de comer, cortar o cocinar. Incluso si el producto se pelará, primero debe lavarse

Frote alimentos firmes, como melones y pepinos, con un cepillo de alimentos limpios Seque el alimento con un paño limpio o una toalla de papel

Mantenga la cocina y su entorno limpio y seguro:

- Lávese las manos, lave cuchillos y tablas de picar después de manipular y preparar alimentos no cocidos

Recuerde que Listeria monocytogenes puede crecer en alimentos en el refrigerador. La temperatura del refrigerador debe estar a $4,4^{\circ} \mathrm{C}$ o menos y del congelador a $-17,7^{\circ} \mathrm{C}$ o menos

Limpie todos los derrames en su refrigerador de inmediato, especialmente jugos de paquetes de salchichas, carne cruda y carne de ave cruda

Cocine las carnes de res y de ave a fondo:

- Cocine a fondo alimentos crudos de origen animal, como carne de res, cerdo o aves de corral, a una temperatura interna segura

Use alimentos precocinados o listos para comer tan pronto como pueda. No guarde el producto en el refrigerador más allá de su fecha de vencimiento

Use las sobras dentro de 3 a 4 días

Utilice alimentos seguros:

No beba leche cruda (no pasteurizada) y no consuma alimentos que usen leche no pasteurizada

Recomendaciones dietéticas para individuos con alto riesgo de listeriosis (mujeres gestantes, pacientes inmunosuprimidos, adultos mayores):

Carnes

No ingiera salchichas, fiambres, embutidos, carnes frías (mortadela), embutidos fermentados o secos a menos que se calienten a una temperatura interna de $74^{\circ} \mathrm{C}$ o hasta que estén calientes antes de servir

Evite que salgan líquidos de los paquetes de salchichas y comida a otros alimentos, utensilios y superficies de preparación de alimentos, y lávese las manos después de manipular salchichas, embutidos y fiambres

No coma paté refrigerado ni productos cárnicos de un mostrador o de la sección refrigerada de una tienda. Los alimentos que no necesitan refrigeración, como los patés enlatados o estables en manteca y carne, son seguros para comer. Refrigérelos después de abrir

Quesos

No coma queso blando como queso feta, queso blanco, queso fresco, queso brie, Camembert, vena azul o panela (queso panela) a menos que esté etiquetado como hecho con leche pasteurizada. Asegúrese de que la etiqueta diga "HECHO CON LECHE PASTEURIZADA"

Tenga en cuenta que el queso elaborado con leche pasteurizada, como el queso de estilo mexicano, que probablemente haya estado contaminado durante la elaboración del queso ha causado listeriosis

Pescados

No consuma mariscos ahumados refrigerados, a menos que estén en un plato cocinado, como una cazuela, o a menos que sea un producto enlatado o de larga duración

Conservas de atún, salmón y otros productos pesqueros conservados y no perecederos son seguros para comer

Melones

Lávese las manos con agua tibia durante al menos 20 seg antes y después de manipular un melón

Frote la superficie de los melones con un cepillo limpio debajo del agua corriente y séquelos con un paño limpio o una toalla de papel antes de cortarlos. Asegúrese de que su cepillo esté desinfectado después de cada uso

Consuma inmediatamente el melón cortado o refrigérelo rápidamente. Mantenga su melón cortado refrigerado por no más de 7 días

Deseche los melones cortados que están a temperatura ambiente por más de $4 \mathrm{~h}$ refrigerada y pescados ahumados, ya que estos alimentos pueden tener un elevado nivel de contaminación ${ }^{70}$. En la Tabla 1 se detalla las medidas de prevención para evitar las infecciones causadas por L. monocytogenes.

El control de listeriosis requiere políticas de salud pública, así como de la industria de alimentos. Por otra parte, el incremento en el uso de medicamentos inmunosupresores para el manejo de enfermedades reumatológicas, malignas o en el trasplante de órganos, ha incrementado la población inmunosuprimida que está en mayor riesgo de listeriosis. Los cambios en la producción de alimentos, uso de refrigeradores, otras tecnologías que permiten el almacenamiento de alimentos por períodos prolongados y el consumo de alimentos no cocidos han incrementado el riesgo de adquirir listeriosis; en consecuencia, se debe tener en cuenta estas recomendaciones para prevenir la infección por L. monocytogenes dado que presenta una elevada mortalidad en comparación con otras enfermedades transmitidas por alimentos. La educación es una de las principales estrategias a adoptar para reducir la incidencia de listeriosis. Otro punto a considerar es que la listeriosis no es una enfermedad de declaración obligatoria en muchos países, por lo que la incidencia real podría ser mayor que la informada ${ }^{22}$.

\section{Conclusiones}

La infección por L. monocytogenes es una realidad en nuestros países, aunque la incidencia y el verdadero impacto de esta enfermedad transmitida por alimentos está subestimada. El profesional de la salud debe conocer los factores de riesgo, las formas de transmisión y reconocer a tiempo a los pacientes con sospecha de infección, sobre todo a los pacientes inmunosuprimidos, mujeres embarazadas y los que se encuentran en los extremos de la vida, debido a la elevada mortalidad que puede tener la listeriosis en este grupo de pacientes. El poder sospechar la enfermedad tempranamente, permitirá realizar un tratamiento empírico inicial que puede modificar el pronóstico, para lo cual la tinción de Gram y los cultivos son fundamentales para identificar a esta bacteria. Así mismo, en muchos países de Latinoamérica se debe establecer mecanismos eficientes de control de alimentos, así como educar a la población general y sobre todo a la población de alto riesgo en las estrategias de prevención de esta enfermedad que puede tener resultados fatales.

\section{Resumen}

La listeriosis es una infección infrecuente pero potencialmente grave, causada por Listeria monocytogenes. La principal vía de transmisión es por el consumo de alimentos contaminados, afecta generalmente a personas 
mayores, mujeres embarazadas y hospederos inmunosuprimidos, aunque también se ven casos en adultos y niños inmunocompetentes. Listeria monocytogenes es un bacilo grampositivo corto, anaerobio facultativo, no formador de esporas, móvil, que provoca una zona angosta de hemólisis en agar sangre. Es un patógeno intracelular facultativo, por lo que presenta una compleja patogenia. Esta bacteria tiene la habilidad de atravesar la barrera intestinal, la placenta y la barrera hemato-encefálica produciendo cuadros de gastroenteritis, infecciones materno-fetales y meningoencefalitis. Se diagnostica, generalmente, a partir de un cultivo positivo de un sitio estéril. El tratamiento de elección incluye el uso de ampicilina intravenosa sola o en combinación con gentamicina.

\section{Referencias bibliográficas}

1.- Murray E G D, Webb R A, Swann M B R. A disease of rabbits characterized by a large mononuclear leucocytosis, caused by a hitherto undescribed bacillus: Bacterium monocytogenes (n.sp.). J Pathol Bacteriol 1926; 29: 407-39.

2.- Lee J, Kyoung W, Hee Nam Ch, Ho Jung M, Han Kang J, Kyu Suh. A case of meningoencephalitis caused by Listeria monocytogenes in a healthy child. Korean J Pediatr 2010; 53 (5): 653-6. doi: 10.3345/ kjp.2010.53.5.653.

3.- Aureli P, Fiorucci G, Caroli D, Marchiaro G, Novara O, Leone L, et al. An outbreak of febrile gastroenteritis associated with corn contaminated by Listeria monocytogenes. N Engl J Med 2000; 342: 1236-41. DOI: 10.1056/ NEJM200004273421702.

4.- Peer M A, Nasir R A, Kakru D K, Fomda B A, Wani M A, Hakeem Q N. Listeria monocytogenes meningoencephalitis in an immunocompetent, previously healthy 20-month old female child. Indian J Med Microbiol 2010; 28 (2): 169-71. http://www. ijmm.org/text.asp?2010/28/2/169/62500.

5.- Dilber E, Aksoy A, Cakir M, Bahat E, Kamasak T, Dilber B. Listeria monocytogenes meningitis in two immunocompetent children. Ann Trop Paediatr 2009; 29 (3): 225-9. doi: 10.1179/0272 49309X12467994694058.

6.- Watson R. Listeriosis remains a cause for concern in Europe. Br Med J 2009; 338: b319. doi: 10.1136/bmj.b319.

7.- López L, Gaztelurrutia L, Martínez I, Aguirrebengoa K, Valls A, Hernández J L, et al. Listeriosis. A 16-year survey in a tertiary hospital in Spain. Rev Panam Infectol 2007; 9 (2): 30-7.

8.- Remington J, Klein J. Infectious Diseases of the Fetus and Newborn Infant. $5^{\text {th }}$ ed.2001; 1157-77.

9.- Little C, Amar C, Awofisayo A, Grant K. Hospital-acquired listeriosis associated with sandwiches in the UK: a cause for concern. $\mathrm{J}$ Hosp Infect 2012; 82: 13-18. doi: 10.1016/j. jhin.2012.06.011.

10.- Benadof D. Listeria monocytogenes. Rev Chilena Infect 2008; 25 (5): 350. http://dx.doi. org/10.4067/S0716-10182008000500005.

11.- Moledo de Vasconcelos R, Castro A,
Ernesto Hofer E, Matias da Silva M, Marín V. Multiplex-PCR serotyping of Listeria monocytogenes isolated from human clinical specimens. Mem Inst Oswaldo Cruz 2008; 103 (8): 836-8. http://dx.doi.org/10.1590/S007402762008000800016.

12.- Baltimore R. Listeria monocytogenes. En: Behrman R, Kliegman R, Jenson H. Nelson Tratado de Pediatría. 16ta ed. McGraw-HillInteramericana, 2000; 900-903.

13.- Rossi M, Paiva A, Tornese M, Chianelli S, Troncoso A. Brotes de infección por Listeria monocytogenes: Una revisión de las vías que llevan a su aparición. Rev Chilena Infect 2008; 25(5): 328-35. http://dx.doi.org/10.4067/S071610182008000500002.

14.- Chaves C, Arias M. Caracterización de cepas de Listeria monocytogenes realizados a partir de queso fresco proveniente de diferentes zonas productoras costarricenses. Arch Latinoam Nutrición 2009; 59 (1): 66-70. https://www. alanrevista.org/ediciones/2009/1/art-10/

15.- Frye D, Zweig R, Sturgeon J, Tormey M, LeCavalier M, Lee I, et al. An Outbreak of febrile gastroenteritis associated with delicatessen meat contaminated with Listeria monocytogenes. Clin Infect Dis 2002; 35: $943-$ 9. DOI: $10.1086 / 342582$.

16.- Varma J, Samuel M, Marcus R, Hoekstra R, Medus C, Segler S, et al. Listeria monocytogenes infection from foods prepared in a commercial establishment: a case- control study of potential sources of sporadic illness in the United States. Clin Infect Dis 2007; 44: 521-8. https://www.jstor.org/ stable/4464032.

17.- Schuchat A, Deaver K, Wenger J D, Plikaytis B D, Mascola L, Pinner R W, et al. Role of foods in sporadic listeriosis.I: Case-control study of dietary risk factors. JAMA 1992; 267: 2041-5. PMID: 1552639.

18.- Ramírez L, Morón de Salim A, Alfieri A, Gamboa O. Frecuencia de Listeria monocytogenes en muestras de tomates (Lycopersicum esculentum) y cilantro (Coriandrum sativum) frescos en tres supermercados de Valencia, Venezuela. Arch Latinoam Nutrición 2009; 59 (3): 318-24. https://www.alanrevista.org/ediciones/2009/3/ art-13/

19.- Torres-Vitela M R, Mendoza-Bernardo
M, Castro-Rosas J, Gómez-Aldapa C A, et al. Incidence of Salmonella, Listeria monocytogenes, Escherichia coli O157:H7, and Staphylococcal enterotoxin in two types of Mexican fresh cheeses. J Food Prot 2012; 75 (1): 79-84. doi: 10.4315/0362-028X.JFP-11258.

20.- Sant'Ana A S, Igarashi M C, Landgraf M, Destro M T, Franco B D. Prevalence, populations and pheno-and genotypic characteristics of Listeria monocytogenes isolated from ready-to-eat vegetables marketed in São Paulo, Brazil. Int J Food Microbiol 2012; 155 (1-2): 1-9. doi: 10.1016/j. ijfoodmicro.2011.12.036.

21.- Hernández-Milian A, Payeras-Cifre A. What is new in Listeriosis? BioMed Res Intern 2014; 1-7. http://dx.doi.org/10.1155/2014/358051

22.- Committee Opinion $N^{\circ} 614$ : Management of pregnant women with presumptive exposure to Listeria monocytogenes. Obstet Gynecol 2014; 124 (6): 1241-4. doi: 10.1097/01. AOG.0000457501.73326.6c.

23.- MacDonald P, Whitwam R, Boggs J, MacCormac J, Anderson K, Reardon J, et al. Outbreak of Listeriosis among Mexican Immigrants as a result of consumption of illicitly produced Mexican-style cheese. Clin Infect Dis 2005; 40: 677-82. DOI: $10.1086 / 427803$

24.- Janakiraman V. Listeriosis in pregnancy: diagnosis, treatment, and prevention. Rev Obstet Gynecol 2008; 1 (4): 179-85. https:// www.ncbi.nlm.nih.gov/pmc/articles/ PMC2621056/pdf/RIOG001004_0179.pdf

25.- Vélez-Leal J L, Dávila-Ramírez F. Listeriosis neonatal en Colombia... iIgual que hace 20 años? Rev Cienc Salud. 2015; 13(2): 301-8. DOI: http://dx.doi.org/10.12804/ revsalud13.02.2015.11.

26.- Koopmans M M, Bijlsma M W, Brouwer M C, van de Beek D, van der Ende A. Listeria monocytogenes meningitis in the Netherlands, 1985-2014: A nationwide surveillance study. J Infect 2017; 75 (1): 12-9. doi: 10.1016/j. jinf.2017.04.004.

27.- Solorzano F, Miranda M. Infección por Listeria monocytogenes. En: González N, Saltigeral P, Macías M. Infectología Neonatal. D.F. México: McGraw-Hill, 2006; 246-50.

28.- Lamont R, Sobel J, Mazaki-Tovi S H, 
Kusanovic J P, Vaisbuch E, Kim S K, et al. Listeriosis in human pregnancy: a systematic review. J. Perinat. Med. 2011; 39: 227-36. doi: 10.1515/JPM.2011.035.

29.- Patil A B, Nadiger S, Chandrasekhar M R, Halesh L H, Kumar M. Listeria monocytogenes meningitis: an uncommon opportunistic infection in HIV/AIDS. Indian J Pathol Microbiol 2007; 50(3): 671-3. PMID: 17883181.

30.- Goulet V, Hedberg C, Le Monnier A, de Valk $\mathrm{H}$. Increasing Incidence of listeriosis in France and other European countries. Emerg Infect Dis 2008; 14 (5): 734-40. doi: 10.3201/ eid1405.071395.

31.- Lewis G, Schweig M, Guillén-Pinto D, Rospigliosi M L. Meningitis neonatal en un hospital general de Lima, Perú, 2008 al 2015. Rev Peru Med Exp Salud Pública 2017; 34 (2): 233-8. doi: 10.17843/rpmesp.2017.342.2297.

32.- Sedano R, Fica A, Guiñez D, Braun S, Porte L, Dabanch J, et al. Infecciones por Listeria monocytogenes, una experiencia de dos décadas. Rev Chilena Infectol 2013; 30 (4): 417-25. http://dx.doi.org/10.4067/S071610182013000400011

33.- Castañeda-Ruelas G, Eslava-Campos C, Castrodel Campo N, León-Félix J, Chaidez-Quiroz C. Listeriosis en México: importancia clínica y epidemiológica. Salud Publica Mex 2014; 56: 654-9. http://www.scielo.org.mx/pdf/spm/ v56n6/v56n6a16.pdf.

34.- Rolhion N, Cossart P. How the study of Listeria monocytogenes has led to new concepts in biology. Future Microbiol 2017; 12 (7): 621-38. doi: $10.2217 / \mathrm{fmb}-2016-0221$

35.- Camejo A, Carvalho F, Reis O, Leitão E, Sousa S, Cabanes D. The arsenal of virulence factors deployed by Listeria monocytogenes to promote its cell infection cycle. Virulence 2011; 2 (5): 379-94. doi: 10.4161/viru.2.5.17703.

36.- Jiwani S H, Wang Y, Dowd G, Gianfelice A, Pichestapong P H, Gavicherla B, et al. Identification of components of the host type IA phosphoinositide 3-kinase pathway that promote internalization of Listeria monocytogenes. Infect Immun 2012; 80 (3): 1252-66. doi: 10.1128/IAI.06082-11.

37.- Struthers J, Westran R. Clinical Bacteriology. ASM Press 2003; 19-31.

38.- Noriega L, Ibáñez S, González P, Yamamoto M, Astudillo J, González M, et al. Listeria monocytogenes: Informe de un aumento de casos en mujeres embarazadas y revisión de la literatura. Rev Chilena Infect 2008; 25 (5): 342-9. http://dx.doi.org/10.4067/S071610182008000500004

39.- Lamond N, Freitag N. Vertical transmission of Listeria monocytogenes: Probing the balance between protection from pathogens and fetal tolerance. Pathogens 2018; 7 (52): 1-14. doi: 10.3390/pathogens7020052.
40.- Arora N, Sadovsky Y, Dermody T, Coyne C. Microbial vertical transmission during human pregnancy. Cell Host Microbe 2017; 21: 561-7. doi: 10.1016/j.chom.2017.04.007.

41.- Allerberger F, Wagner M. Listeriosis: a resurgent foodborne infection. Clin Microbiol Infect 2010; 16: 16-23. doi: 10.1111/j.14690691.2009.03109.x.

42.- Kimberlin D W, Brady M T, Jackson M A, Long S S. Red Book: Report of the Committee on Infectious Diseases. 30th ed. Elk Groove Village, IL, American Academy of Pediatrics, 2015; 513-6.

43.- Flickinger Jr J, Rodeck U, Snook A. Listeria monocytogenes as a vector for cancer immunotherapy: Current Understanding and Progress. Vaccines. 2018; 6(48): 1-19. doi: 10.3390/vaccines6030048.

44.- Miranda G, Orellana P, Dellien H Z, Switt M. Romboencefalitis por Listeria monocytogenes. Patología emergente en relación al brote epidémico. Presentación de tres casos clínicos. Rev Med Chile 2009; 137 : 1602-6. http://dx.doi.org/10.4067/S003498872009001200009.

45.- Mansbridgea Ch, Grecua I, Li Voon Chonga J, Vanderveldea C, Saeeda K. Two cases of listeria rhombencephalitis. ID Cases 2018; 11: 22-5. https://dx.doi.org/10.1016\%2Fj. idcr.2017.12.002.

46.- Laciar A, Vaca Ruiz M, Monnier A. Neonatal Listeria-meningitis in San Luis, Argentina: a three-case report. Rev Argent Microbiol 2011; 43: 45-7. doi: 10.1590/S032575412011000100010 .

47.- Tourret J, Benabdellah N, Drouin S, Charlotte F, Rottembourg J, Arzouk N, et al. Unique case report of a chromomycosis and Listeria in soft tissue and cerebellar abscesses after kidney transplantation. BMC Infect Dis 2017; 17: 288. doi: 10.1186/s12879-017-2386-9.

48.- Sia C, Wilson S, Ananda-Rajah M, Mills J, Aung AK. Listeria monocytogenes peritonitis in an HIV-infected patient. Clin Nephrol 2017; 87 (5): 267-70. doi: 10.5414/CN108897.

49.- Le Monnier A, Blanot S, Abachin E, Beretti J, Berche P, Kayal S. Listeria monocytogenes: a rare complication of ventriculoperitoneal shunt in children. J Clin Microbiol 2011; 49 (11): 3924-7. doi: 10.1128/JCM.01083-11.

50.- Thompson A, Wrubel D, Berkowitz F. Grampositive rods on a cerebrospinal fluid Gram stain. Open Forum Infect Dis 2017; 4 (4): ofx240. doi: 10.1093/ofid/ofx240.

51.- Liu D, Wang Y, Wang Y, Zhang L, Luo L, Liu K, et al. Development of a novel Listeria enrichment broth for the isolation of pathogenic Listeria. J Food Prot 2017; 80 (10): 1768-76. doi: 10.4315/0362-028X.JFP-16-52.

52.- Shum D, Galsworthy S. Stimulation of monocyte production by an endogenous mediator induced by a component from Listeria monocytogenes. Immunology 1982; 46: 343-51. PMID: 6806176.

53.- Heger W, Dierich M P, Allerberger F. In vitro susceptibility of Listeria monocytogenes: comparison of E-test with agar dilution test. Chemotherapy 1997; 43: 303-10. DOI: $10.1159 / 000239582$.

54.- Conter M, Paludi D, Zanardi E, Ghidini S, Vergara A, Ianieri A. Characterization of antimicrobial resistance of foodborne Listeria monocytogenes. Int J Food Microbiol 2009; 128: 497-500. doi: 10.1016/j. ijfoodmicro.2008.10.018.

55.- Pagliano P, Arslan F, Ascione T. Epidemiology and treatment of the commonest form of listeriosis: meningitis and bacteraemia. Infez Med. 2017; 3: 210-6. https://www.infezmed.it/ media/journal/Vol_25_3_2017_2.pdf.

56.- Tuazon C, Shamsuddin D, Miller H. Antibiotic susceptibility and synergy of clinical isolates of Listeria monocytogenes. Antimicrob Agents Chemother 1982; 21 (3): 525-7. PMCID: PMC181930.

57.- Azimi P H, Koranyi K, Lindsey K D. Listeria monocytogenes: synergistic effects of ampicillin and gentamicin. Am J Clin Pathol 1979; 72 (6): 974-7. PMID: 117697.

58.- Polat M, Kara S S, Tapısız A, Derinöz O, Çağlar K, Tezer H. Successful treatment of refractory listeria meningitis and bacteremia with trimethoprim-sulfamethoxazole in an immunocompetent child. Turk J Pediatr 2016; 58: 220-2. DOI: 10.24953/ turkjped.2016.02.017.

59.- Moura Falavina dos Reis C, Barbosa A, Alves Rusak L, Vallim D, Hofer E. Antimicrobial susceptibilities of Listeria monocytogenes human strains isolated from 1970 to 2008 in Brazil. Rev Soc Bras Med Trop 2011; 44(2): 173-6. http://dx.doi.org/10.1590/S003786822011005000019

60.- Ruiz-Bolivar Z, Neuque-Rico M C, PoutouPiñales R A, Carrascal-Camacho A K, Mattar S. Antimicrobial susceptibility of Listeria monocytogenes food isolates from different cities in Colombia. Foodborne Pathog Dis 2011; 8 (8): 913-9. doi: 10.1089/fpd.2010.0813.

61.- Rodas-Suárez O, Flores-Pedroche J, Betancourt-Rule J, Quiñones-Ramírez E, Váquez-Salinas C. Occurrence and antibiotic sensitivity of Listeria monocytogenes strains isolated from oysters, fish, and estuarine water. Appl Environ Microbiol 2006; 72 (11): 7410-2. DOI: 10.1128/AEM.00956-06.

62.- Chan L-M, Lin H-H, Hsiao Sh-M. Successful treatment of maternal Listeria monocytogenes bacteremia in the first trimester of pregnancy: A case report and literature review. Taiwan J Obstet Gynecol 2018; 57: 462-3. DOI: 10.1016/j.tjog.2018.04.025.

63.- Awofisayo A, Amar C, Ruggles R, Elson R, Adak G K, Mook P, et al. Pregnancy-associated 
listeriosis in England and Wales. Epidemiol. Infect 2015; 143: 249-56. doi: 10.1017/ S0950268814000594.

64.- Grayo S, Join-Lambert O, Desroches M C, Le Monnier A. Comparison of the in vitro efficacies of moxifloxacin and amoxicillin against Listeria monocytogenes. Antimicrob Agents Chemother 2008; 52: 1697-702. doi: 10.1128/AAC.01211-07.

65.- Morosi S, Francisci D, Baldelli F. A case of rhombencephalitis caused by Listeria monocytogenes successfully treated with linezolid. J Infect 2006; 52: 73-5. DOI: 10.1016/j.jinf.2005.06.012.

66.- Pupo I, Lepe J A, Smani Y, Aznar J. Comparison of the in vitro activity of ampicillin and moxifloxacin against Listeria monocytogenes at achievable concentrations in the central nervous system. J Med Microbiol 2017; 66 (6): 713-20. doi: 10.1099/jmm.0.000486.

67.- Muanda F T, Sheehy O, Bérard A.

Use of antibiotics during pregnancy and the risk of major congenital malformations: a population based cohort study. $\mathrm{Br} \mathrm{J}$ Clin Pharmacol 2017; 83 (11): 2557-71. doi: $10.1111 /$ bcp. 13364 .

68.- Hakenbeck R, Hof H. Relatedness of penicillinbinding proteins from various Listeria species. FEMS Microbiol Lett 1991; 84: 191-6. https:// doi.org/10.1111/j.1574-6968.1991.tb04595.x.
69.- Escolar C, Gómez D, Rota García M, Conchello P, Herrera A. Antimicrobial resistance profiles of Listeria monocytogenes Listeria innocua and Isolated from ready-to-eat products of animal origin in Spain. Foodborne Pathog Dis. 2017; 14 (6): 357-63. doi: 10.1089/ fpd.2016.2248.

70.- Jacobson L. Listeriosis. Pediatr Rev 2008; 29: 410-1. doi: 10.1542/pir.29-11-410.

71.- American Academy of Pediatrics, Committee on Infectious Diseases and Committee on Nutrition. Consumption of raw or unpasteurized milk and milk products by pregnant women and children. Pediatrics 2014; 133: 175. doi: 10.1542/peds.2013-3502. 